\title{
Heavy Ion Beam Probe, Present Status and Future Development
}

\author{
Y. HAMADA, A. NISHIZAWA, T. WATARI, K. NARIHARA, Y. KAWASUMI, T. IDO, M. KOJIMA, \\ K. TOI and JIPPT-IIU Group \\ National Institute for Fusion Science, Toki 509-5292, Japan
}

(Received 26 December 2006 / Accepted 19 April 2007)

\begin{abstract}
Present status of the analysis of HIBP data on zonal flows, streamers and drift-wave turbulence of the JIPPTIIU tokamak plasmas, is reviewed. Limiting factors and improvements of heavy ion beam probe towards better understanding of tokamak turbulence, beam attenuation, beam quality of the probing beam, multiple energy analyzers for simultaneous measurement at various cross-sections of the plasmas, proposal for 2-dimensional measurement of the plasma turbulence, are discussed.
\end{abstract}

(C) 2007 The Japan Society of Plasma Science and Nuclear Fusion Research

Keywords: diagnostic beam, drift wave, turbulence, zonal flow, streamer

DOI: $10.1585 /$ pfr.2.S1024

\section{Introduction}

Heavy ion beam probe (HIBP) is a unique and powerful diagnostics, since it provides a local and nonperturbative measurement of plasma potential $(\Phi)$. HIBP is the ideal diagnostics for the detection of zonal flow as well as streamers, since the sensitivity of small zonal flow as slow as ion diamagnetic drift velocity can be detected with high signal/noise ratio through $-\nabla \Phi \times B / B^{2}$ drift. As for the locality, it can be a Langmuire probe in the hot plasma core. However, the recent analysis of zonal flow and streamer in JIPPT-IIU HIBP clarifies the necessity of many modifications of the present HIBP for the future study of the turbulence in tokamak plasmas. One of the most serious problem may be smallness of the penetration length for core plasma, of the present $\mathrm{Tl}^{+}, \mathrm{Cs}^{+}$, or $\mathrm{Au}^{+}$ injection beam species. In order to increase the penetration depth, we use $\mathrm{Na}^{+}$beam instead of $\mathrm{Tl}^{+}$beam. We discuss its successful results and make a survey of species of the beam for future experiment.

\section{Present Status of Analysis of Toka- mak Turbulence, Zonal Flows and Streamers}

\subsection{Short history}

A. Hasegawa et al. pointed out in 1979 that the driftwave turbulence should induce zonal flow along the magnetic surface, and zonal flows would have stabilizing effect by the shearing of the drift-wave by the gradient of the flow velocity [1]. In 1987, A. Hasegawa et al. showed in the computer simulation of the cylindrical plasma, the generation of zonal flows along the magnetic surface by $E_{r} / B_{t}$ through the buildup of the potential across the magnetic surfaces [2]. In 1988 J.F. Drake pointed out that slab plasmas have sharp structures, very narrow in poloidal di- rections and wide in radial direction by the buildup of the potential difference along poloidal direction and called this flow as streamers [3]. Later streamers and zonal flows are found to be fundamental phenomena in the large-scale computer simulation of tokamak plasmas [4-11].

Experimentally, early in $1990 \mathrm{~s}$, HIBP measurements in TEXT and JIPPT-IIU tokamak $[12,13]$ found that the secondary beam has large energy change due to local electric potential or the acceleration by the magnetic fluctuation, in the tokamak plasma column up to a few hundred electron Volt peak to peak in tens of $\mathrm{kHz}$ frequency regions with poloidal mode number (m), $m=0$ [14]. We and probably TEXT group, at first, doubted that these oscillations may be noise induced by many high-voltage dc power supplies (HVPSs) in the HIBP. The HIBP has about 10 or more HVPSs for the ion source, acceleration to about half of million Volts, focusing by electric quadrupole lenses, deflection, sweeping of the beam across the cross-section of plasma column and high-voltage energy analyzer for secondary beam. Every highly stabilized HVPS rectifies the output of $30-50 \mathrm{kHz}$ high-voltage oscillators, since the smoothing action by network of capacitor and inductance is very effective in this frequency range compared to 50 or $60 \mathrm{~Hz}$. This frequency range is in rough agreement with that of the observed energy change of the secondary beam in the tokamak plasmas. We reduced ripples of HVPS by inserting the filter network and concluded that the energy change are due to plasma instead of being induced by ripples in power supplies. The most conclusive experiment may be the measurement of potential in the tokamak vessels by HIBP when the toroidal electric field for the plasma startup is not applied. The helium gas ionizes injected heavy ion beam and we found that there is no large energy change in this case $[15,16]$.

The TEXT groups suggested that the drift-tearing mode $(m=10-20)$ of the tokamak plasmas causes these 


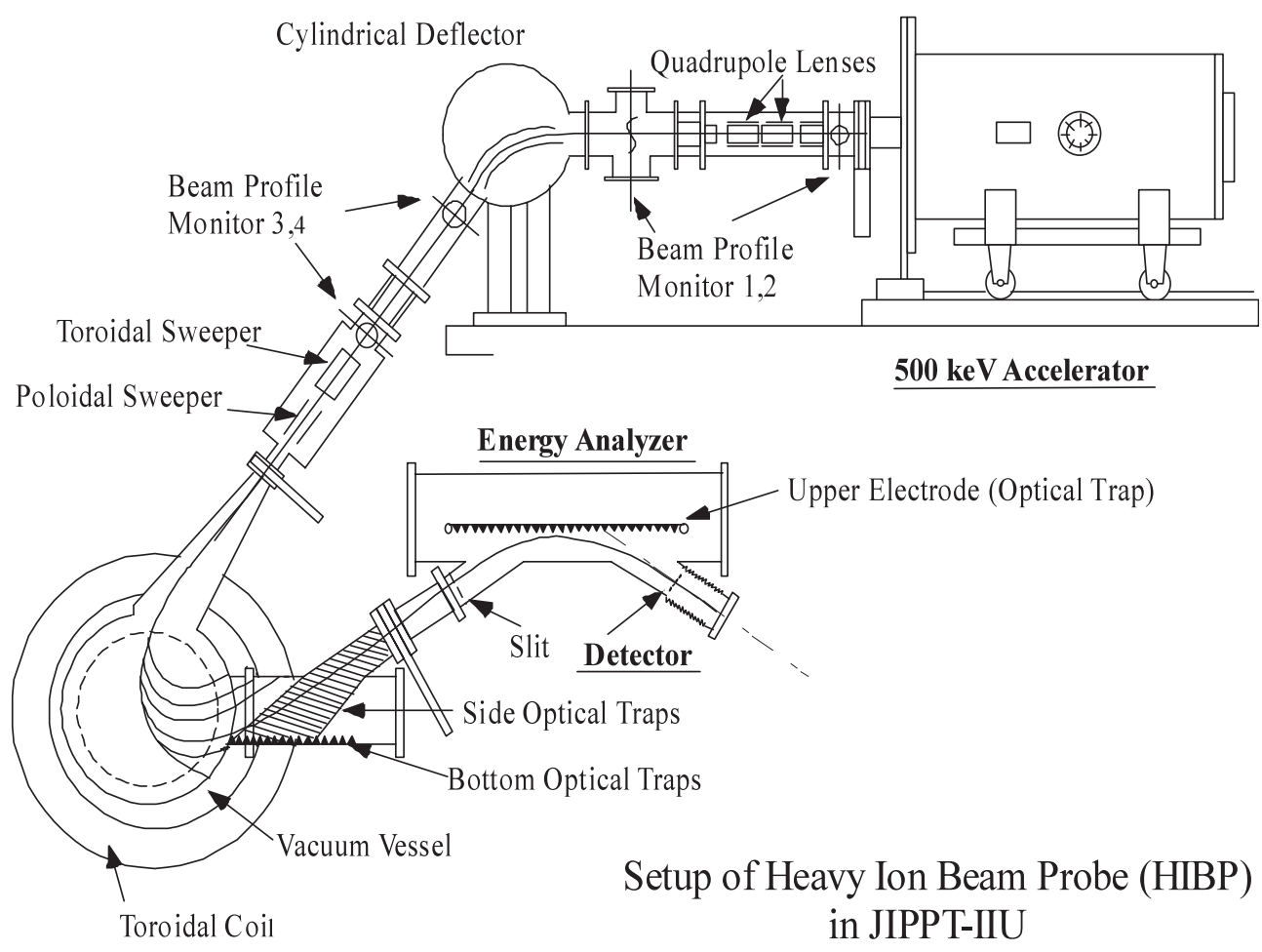

(a)

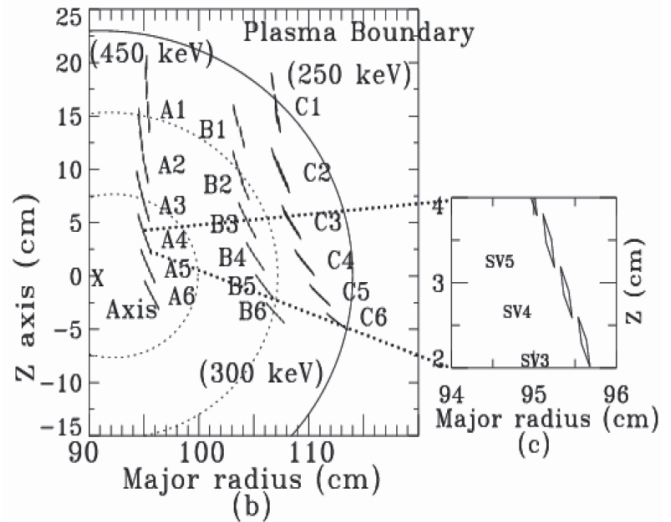

Fig. 1 (a) The setup of heavy ion beam probe at JIPPT-llU tokamak plasmas. (b) Locations of 6 sample volumes of 6 steps at 3 different beam energies of JIPP T-1lU HIBP. The injected beam was swept in steps (A1-A6 $₫$ B1-B6 and C1C6) by changing the injection angle. The label A stands for the beam energy of $450 \mathrm{keV}$, label $\mathrm{B}$ for $300 \mathrm{keV}$ and label $\mathrm{C}$ for $250 \mathrm{keV}$. In this example the toroidal magnetic field is assumed to be 3 Tesla. The dashed circles show locations of magnetic surfaces at $1 / 3$ and $2 / 3$ of the minor radius of $23 \mathrm{~cm}$. The outer shift of the center is calculated by the assumption that the current profile is $\mathrm{T}_{e}(\mathrm{r})^{(3 / 2)} \mathrm{de}-$ termined by Thomson scattering method and $\beta_{p}=0$. (c) Expanded view of the sample volumes at step A4. From Y. Hamada et al., Plasma Phys. Control. Fusion 48, S177 (2006).

phenomena since the HIBP is very susceptible to these MHD phenomena [14]. The micro-magnetic field induces the additional deflections of the secondary beam into toroidal direction or acceleration by magnetic fluctuations.
This deflection can be measured as the energy change in the parallel plate energy analyzer, since this energy analyzer measures the energy of the secondary beam in the analyzer plane perpendicular to the parallel plate and slit/detector plane. We measured the energy of the secondary beam by changing analyzer plane a little by little and concluded that the energy change is not due to the deflection of the beam. We reported in 1995 at seventh international Toki conference that the large energy change in the core is pure potential fluctuations or the acceleration of the beam by the change of magnetic vector potential $[15,16]$. In addition, to interpret this difference of the facts that the poloidal mode number $m$ is zero and the drifttearing mode of $\mathrm{m}$ is larger than 10 , is very difficult.

In 1996, we concluded in the study of observed large energy change at the sawtooth crash [17] that the acceleration of the HIBP beam by the magnetic fluctuation through the time derivative of vector potential is unlikely, because low- $\beta$ plasma like low-density $\mathrm{OH}$ plasmas have vector potential parallel to the main magnetic field [18] and the trajectory of HIBP beam is perpendicular to the magnetic field. Accordingly the large energy change of the beam means the existence of large electro-static potential oscillations in the plasma. The existence of zonal flows was already discussed in the large-scaled simulation of the tokamak plasmas when we found the large potential oscillations in the tokamak plasmas. But at that time zonal flow phenomena is thought to be low-frequency and rather stationary instead of the $10-50 \mathrm{kHz}$ phenomena. Eight years later TEXT group suggested this is the potential oscillation [19]. 
In 2003, McKee et al., pointed out that the time behavior of local density measure by BES (beam emission spectroscopy) in the DIII-D can be interpreted to be the poloidal rotation of the plasma column and the rotational velocity is modulated strongly by $10-20 \mathrm{kHz}$ oscillations [20]. They ascribed these modulations to the geodesic acoustic mode (GAM) [21] excited in the tokamak plasma. We started to analyze in detail the large potential oscillations in the tokamak plasma since our measurement of local potential gives directly zonal flows and streamers through $-\nabla \Phi \times B / B^{2}$.

\subsection{Zonal flow and streamers in JIPPT-IIU tokamak plasmas}

Figure 1(a) shows the setup of $500 \mathrm{keV}$ HIBP for JIPPT-IIU [22] tokamak which shut down in the fall of 1995 and the position and shape of the sample volumes (SVs) are shown in Fig. 2(b) and 2(c) [23-26]. The power source (HVPS) for the electrostatic acceleration up to $500 \mathrm{keV}$ is originally developed for electron microscopes. The HVPS for electron microscopes has to be highly stabilized since the chromatic aberration of the electron microscope is caused by the ripple of HVPS. Ours HVPS has about $2 \mathrm{Vp}$-p at the voltage of $500 \mathrm{keV}$ [13]. The analyzer has 7 sets of input slit opening and detector plates, about $1 \mathrm{~cm}$ apart each other [23]. The secondary beam to the analyzer tends to be diverging at the analyzer, and out of seven, we can measure at 6 sample volumes (SVs) routinely.

The beam source is a thermionic ion source and its temperature is about $0.1 \mathrm{eV}$ and its size is about $3 \mathrm{~mm}$. In addition, the beam current is about order of $10 \mu \mathrm{A}$ in the experiment. Accordingly the phase-volume of the $500 \mathrm{keV}$ beam is so small, we can focus from about $10 \mathrm{~mm}$ in diameter to the size of about $2 \mathrm{~mm}$ in diameter in the plasma by the focal length of $2 \mathrm{~m}$. The position of the array of $6 \mathrm{SVs}$ can be swept like steps A1 to A6 for $450 \mathrm{keV}$ beam and steps B1-B6 for $300 \mathrm{keV}$ and steps C1-C6 for $250 \mathrm{keV}$ by changing the poloidal injection angle through the poloidal sweeper at the injection port of the primary beam. The shape of SVs is like a rhombus and the longer diagonal of the rhombus is about $5 \mathrm{~mm}$, although its dimensions and shape are different on the position of the steps. These values are listed on Table 1 [24].

Figure 2 shows the time trace of the density and potential at $\mathrm{A} 4$ (core area, $r / a_{p} \cong 0.2$ ), and C2 (outer area, $r / a_{p} \cong 0.8$ ) of the low-density $\mathrm{OH}$ plasmas. At the core area, the fluctuation level of the normalized density fluctuation, $\tilde{n}_{e} / \bar{n}_{e}$, (Fig. $2(\mathrm{~d})$ ), is a few percents, as is discussed by HIBP and BES measurement, while the potential fluctuation (Fig. 2(c)) is very intense and can be about $400 \mathrm{Vp}$ $\mathrm{p}$ [26].

The time traces at $\mathrm{C} 2$ have very different characteristics, the signals tends to have sharp fronts, and $\tilde{n}_{e} / \bar{n}_{e}$ is order of 0.5-1.0 and the potential variation is order of order of $300 \mathrm{eV}$, about the electron temperature at $\mathrm{C} 2$. This fluctuation level is almost the same at steps C1-C6. The sharp fronts are observed at $\mathrm{B} 2$ - $\mathrm{B} 6$ steps $\left(r / a_{p} \cong 0.6\right)$ with small

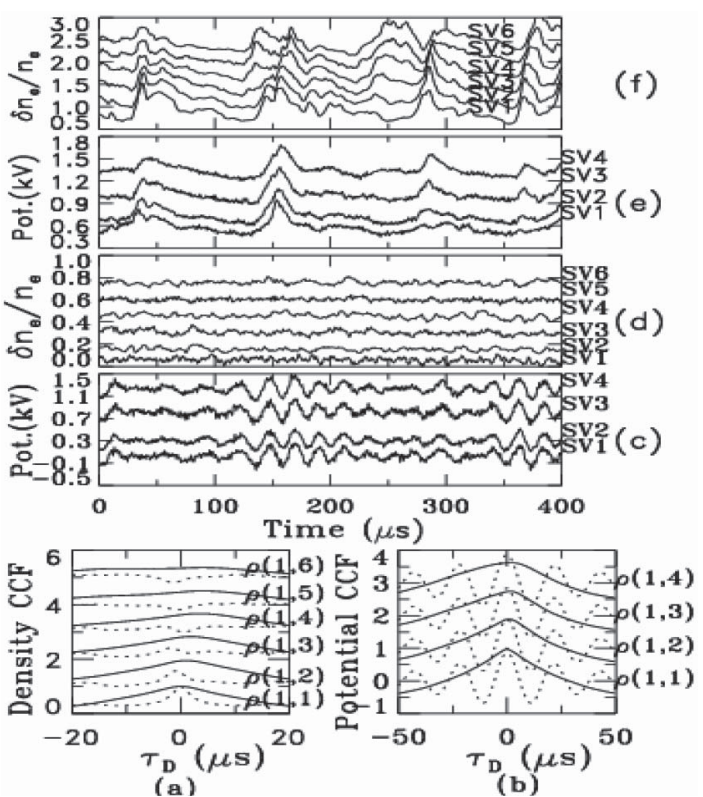

Fig. 2 (a) Correlation coefficient function (CCFs) of density fluctuations at $\mathrm{C} 2$ and at $\mathrm{A} 4$ (dotted line). (b) CCF of potential fluctuations at $\mathrm{C} 2$ and $\mathrm{A} 4$ (dotted curves). (c), (d) Time-expanded raw data of potential, (c) and local density fluctuations, (d) at A4. (e), (f). Raw data for potential, (e) and density fluctuations, (f) at C2. Correlation was taken between signals at $\mathrm{SV} 1$ and $\mathrm{SV}_{j}$ ( $j=1-6$ for density (c) and $j=1-4$ for potential measurement). Correlation coefficient functions (CCFs) for functions $u$ ad $v, \rho\left(u, v, \tau_{d}\right)$, is defined by $\rho\left(u, v, \tau_{d}\right)=$ $C\left(u, v, \tau_{d}\right) / \sqrt{C(u, u, 0)} / \sqrt{C(v, v, 0)}$, where $C\left(u_{i}, v_{j}, \tau d\right)=$ $\int_{-T}^{+T} u_{i}(t) v_{j}\left(t+\tau_{d}\right) d t . T$ is chosen as $2 \mathrm{~ms}$ in this analysis. Multiple curves are displaced upwards to avoid the overwriting. CCFs are displayed by shifting upwards by j-1. From Y. Hamada et al., Phys. Rev. Lett. 96115003 (2006).

amplitude and the potential waveform is mild. We assigned these fluctuations as streamer and discuss it later.

CCF (cross correlation function) of potentials at SV1 of A4, $\Phi_{A, 4,1}$ and $\mathrm{SVm}$ of A4, $\Phi_{A, 4, m}$ are shown in dotted curves of Fig. 2(b). Here CCF of function, $u$ and $v$ is defined by $\rho\left(u, v, \tau_{d}\right)=\frac{C\left(u, v, \tau_{d}\right)}{\sqrt{C(u, u, 0)} \sqrt{C(v, v, 0)}}$ and $C\left(u_{i}, v_{j}, \tau_{d}\right)=$ $\int_{-T}^{+T} u_{i}(t) v_{j}\left(t+\tau_{d}\right) d t$ with $T=2 \mathrm{~ms}$ in this analysis. Potential CCF, $\rho(1, \mathrm{~m}, \tau)$ does not show any shift of the peak as is shown in Fig. 2(b) and we know that poloidal mode number $(m)$ is zero, the high-frequency zonal flow. The density CCFs shown in dotted curves of Fig. 2(a) shows the propagation into electron diamagnetic drift direction and show rather short correlation length of $2 \mathrm{~cm}$ or 3 in poloidal distance. In this sense, the flux tube with diameter of 2 or $3 \mathrm{~cm}$ is independent in terms of the drift-wave turbulence. The potential and density CCFs at C2 (solid curves) are very similar to each other and propagate into ion diamagnetic drift direction.

The radial coherent length of high-frequency zonal flow may be determined by ratio of CCFs in the follow- 
Table 1 The positions $(R(\mathrm{~cm}), \mathrm{Z}(\mathrm{cm}), \mathrm{r}(\mathrm{cm}))$ are shown in the 2nd to 4th columns for each step specified by $S V_{j, k, 1}$ for $\mathrm{j}=\mathrm{A}, \mathrm{B}, \mathrm{C}$, and $\mathrm{k}=1$ to 5 . The 5th column, $r$ denotes the length of the longer diagonal of the rhombus-like shape of $\mathrm{SV}_{\mathrm{j}, \mathrm{k}, 1}$. The 6th column, $\delta \mathrm{r}$ shows the radial separations between the sample volumes $\mathrm{SV}_{\mathrm{j}, \mathrm{k}, 3}$ and $\mathrm{SV}_{\mathrm{j}, \mathrm{k}, 1}$. The 7 th column shows the ration of coherence, $\eta=\left(\rho\left(\Phi_{\mathrm{j}, \mathrm{k}, 3}, \Phi_{\mathrm{j}, \mathrm{k}, 1}\right) / \rho\left(\Phi_{\mathrm{j}, \mathrm{k}, 1}, \Phi_{\mathrm{j}, \mathrm{k}, 1}\right)\right.$, the ration of correlation between potentials at SV3 and SV1 with autocorrelation of potential at SV1. The 8th column shows the radial correlation length $l_{c}$, calculated using $l_{c}=\left|\delta \mathrm{r} / \log \left[\rho\left(\Phi_{\mathrm{j}, \mathrm{k}, 3}, \Phi_{\mathrm{j}, \mathrm{k}, 1}\right) / \rho\left(\Phi_{\mathrm{j}, \mathrm{k}, 1}, \Phi_{\mathrm{j}, \mathrm{k}, 1}\right)\right]\right|$. From Y. Hamada $₫$ Nuclear Fusion 45, 81 (2005).

$\begin{array}{lrrrrrrc}\text { Step } & \mathbf{R}(\mathbf{c m}) & \mathbf{Z}(\mathbf{c m}) & \mathbf{r}(\mathbf{c m}) & \boldsymbol{r l}(\mathbf{c m}) & \boldsymbol{\delta} \mathbf{r}(\mathbf{c m}) & \boldsymbol{\eta} & \boldsymbol{l}_{\boldsymbol{c}} \\ \mathrm{A} 1 & 95.46 & 14.10 & 14.32 & 1.32 & 3.25 & 0.20 & 2.00 \\ \mathrm{~A} 2 & 95.32 & 9.14 & 9.43 & 0.89 & 1.74 & 0.25 & 1.24 \\ \mathrm{~A} 3 & 95.40 & 5.44 & 5.95 & 0.71 & 1.23 & 0.72 & 3.78 \\ \mathrm{~A} 4 & 95.61 & 2.30 & 3.48 & 0.61 & 0.64 & 0.89 & 5.48 \\ \mathrm{~A} 5 & 95.91 & -0.43 & 2.94 & 0.53 & -0.41 & 0.80 & 1.78 \\ \mathrm{~A} 6 & 96.28 & -2.93 & 4.40 & 0.49 & -0.90 & 0.57 & 1.61 \\ \mathrm{~B} 1 & 104.05 & 12.47 & 16.66 & 0.90 & 0.77 & 0.64 & 1.72 \\ \mathrm{~B} 2 & 104.45 & 7.43 & 13.65 & 0.62 & 0.42 & 0.91 & 4.32 \\ \mathrm{~B} 3 & 105.07 & 3.79 & 12.65 & 0.52 & -0.14 & 0.93 & 1.91 \\ \mathrm{~B} 4 & 105.82 & 0.71 & 12.84 & 0.45 & -0.48 & 0.77 & 1.85 \\ \mathrm{~B} 5 & 106.69 & -1.97 & 13.83 & 0.40 & -0.65 & 0.63 & 1.39 \\ \mathrm{~B} 6 & 107.67 & -4.28 & 15.28 & 0.37 & -0.72 & 0.15 & 0.37 \\ \mathrm{C} 1 & 107.30 & 14.23 & 20.18 & 1.64 & 1.04 & 0.80 & 4.66 \\ \mathrm{C} 2 & 108.10 & 8.31 & 17.23 & 1.11 & 0.16 & 0.91 & 1.70 \\ \mathrm{C} 3 & 109.11 & 3.83 & 16.56 & 0.86 & -0.30 & 0.93 & 3.98 \\ \mathrm{C} 4 & 110.32 & 0.33 & 17.33 & 0.73 & -0.53 & 0.76 & 1.91 \\ \mathrm{C} 5 & 111.73 & -2.55 & 18.90 & 0.65 & -0.64 & 0.44 & 0.77 \\ \mathrm{C} 6 & 113.34 & -4.90 & 20.93 & 0.61 & -0.68 & 0.05 & 0.23\end{array}$

ing way.

$$
l_{c}=\left|\delta r / \log \left[\rho\left(\Phi_{\mathrm{j}, \mathrm{k}, 3}, \Phi_{\mathrm{j}, \mathrm{k}, 1}\right) / \rho\left(\Phi_{j, k, 1}, \Phi_{j, k, 1}\right)\right]\right|,
$$

where $\delta r$ is the radial difference between SV3 and SV1 and shown in Table 1. This value is shown in the $8^{\text {th }}$ column of the table 1 and is order of $1 \mathrm{~cm}$ at A to B steps. At step $\mathrm{C}$ the high-frequency zonal flow does not dominate at potential fluctuations and the potential fluctuations tends to have drift-wave characteristics as shown in solid curves of Fig. 2(a) and 2(b).

The radial correlation length tends to have large value near steps A4 and B2 as shown in Table 1. The SV array at $\mathrm{A} 4$ and $\mathrm{B} 2$ are nearly along the magnetic surface since near A4, B2 $\delta r$ changes its sign as shown in Table 1. Accordingly the equation of $l_{c}$ tends to have large error because of zero/zero procedure, the error due to the one-dimensional measurement by HIBP. We need to have the capability for 2-D measurement.

Figure 3 shows the difference of the zonal flow spectra at $\mathrm{OH}$ phase and heating phase. The dominance of highfrequency zonal flows in the GAM frequency range can be clearly observed, as is also apparent at Fig. 2(c) in $\mathrm{OH}$ phase, while we can clearly observe the growth of the lowfrequency zonal flow and the decrease of the intensity of high-frequency zonal flow in the heating phase. This may be induced by the change of trapped electron mode (TEM) plasma of low-density $\mathrm{OH}$ phase to the ion temperature gradient mode (ITG) plasma. Our spectrum of zonal flow

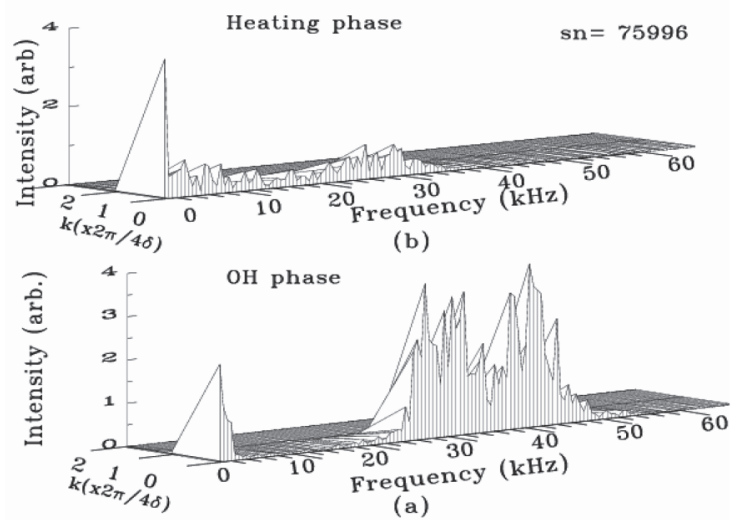

Fig. 3 (a) shows the zonal flow spectrum in $\mathrm{OH}$ low-density plasma phase, (a) and heated plasma phase. (b). From Y. Hamada et al., Phys. Rev. Lett. in press.

in ITG plasma are similar to the one given in the largescale computer simultion by Hahm et al. [27], and we hope in near future we can compare the zonal flow spectrum of TEM plasma calculated by large-scale computer simulations with our spectrum. These changes of zonal flow spectra are very recent topic of the experimental study of zonal flow and our results will soon be printed in Physical Review Letters.

Figure $4(b, c)$ shows time traces of density and potential at $\mathrm{C} 5$, where the array of SVs are rather radial. In this 


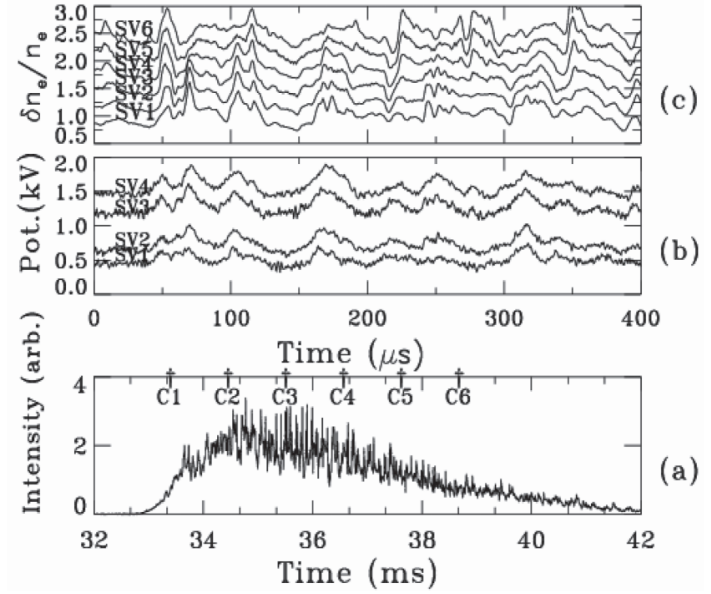

Fig. 4 (a) shows the time behavior of the intensity of the secondary beam, when the beam is swept continuously through C1- C6 intead of steps. Also time expanded view of raw data of density (2b) and potential (2c) at C5 by step mode are shown. From Y. Hamada et al., Phys. Rev. Lett. 96115003 (2006).

case, the characteristics of sharp fronts and large fluctuation level of density and potential are almost the same with the traces at $\mathrm{C} 2$. The difference may be very fast propagation or rather the same phase at the radial direction. The radial coverage of our HIBP is too small and we need to have wide coverage and 2-D measurement to clarify the radial extent and propagation characteristics of streamers. Figure 4(a) shows the intensity of the secondary beam (corresponding to the local density) of SV1 when SVs are swept smoothly from vacuum to C1-C6. Near C1, the SV1 cross the plasma boundary to more dense area of $\mathrm{C} 3$ or $\mathrm{C} 4$, where the electron temperature is about $400 \mathrm{eV}$. We can clearly show that this streamer occurs fairy deep into the plasma. The similar observations were performed at the scrape-off layer and near the last closed magnetic surface $[28,29]$. They are sometimes referred as blobs. We may be able to state that the streamers we found in the outer region of the plasma column, migrate to the edge and become blobs. The electric field between SV1 and SV2, $\left(\Phi_{\mathrm{C}, 2,1}-\Phi_{\mathrm{C}, 2,2}\right)$, and the electric field between SV3 and SV4, $\left(\Phi_{\mathrm{C}, 2,3}-\Phi_{\mathrm{C}, 2,4}\right)$ at $\mathrm{C} 2$, where SVs are aligned nearly along the magnetic surface, are so different that we may be able to conclude the streamer has short poloidal characteristic length of twice the distance between SVs , about $1.3 \mathrm{~cm}$ [26]. This is also in agreement with the prediction of streamers.

\section{Requirement for Further Analysis}

Nowadays, the advantage of having SVs widely aparts may be listed below, as

1) The radial eigen mode of the drift-wave can be wide in radial direction as it is shown in the flow pattern of the eddies of the large-scale computer simulation.
2) The eigen mode pattern of ballooning pattern of the drift wave is not experimentally established because of the lack of simultaneous turbulence measurement at far-apart SVs. 3 ) The radial extent of the streamer is much larger than our coverage of about $3 \mathrm{~cm}$.

4) The radial coherent length of the zonal flow is short, about 1 or $2 \mathrm{~cm}$, the radial coherent length of the density turbulence especially in low-density $\mathrm{OH}$ plasma, can be longer than our coverage, as is shown in our measurement [17].

5) As was done by CHS HIBP [30], the zonal field measurement is particularly useful by multiple and far apart SVs since we can tell easily select the zonal flow utilizing the same potential change on the same magnetic surface.

6) 2-dimensional measurement of the turbulence is useful, since every instability is 2 dimensional eddies and streamer and zonal flow is also 2-dimensional.

HIBP should be able to cover the whole cross-section by simultaneous measurement. Since the coverage of the distance by one energy analyzer is limited as we stated before, the increase of the number of the energy analyzer at one injected beam, is easiest way to handle these problems.

\section{Future Development}

\subsection{Multiple sets of input-slit and detectors, multiple analyzers}

The SVs should be increased as many as possible to have reasonable $k$-spectrum resolution. Since the analyzer is a parallel-plate electrostatic analyzer, we can install several set of input-slit and detectors in the analyzer. We have installed 7sets of the input slits and detectors which are $1 \mathrm{~cm}$ apart each other perpendicular to the incident beam, at the JIPPT-IIU HIBP analyzer. We usually have density measurement at $6 \mathrm{SVs}$ instead of 7, and potential measurement at $5 \mathrm{SVs}$. The limit is due to the different injection angle of the beam let to each input slits, since the secondary beam tends to be diverging beam to the analyzer as we mentioned before. Accordingly, when we want to have much larger coverage for simultaneous measurement of the tokamak turbulence, in the cross-section of the tokamak plasma column, we have to separate the sheet-like secondary beam into several sections which has different injection angle as an average to the analyzer. Then we have to insert a sweeper to each group for the adjustment of the input angle to the analyzer as shown in Fig. 5.

In order to have much wider coverage, installment of several analyzers at different ports of the tokamak can be performed as is also shown in Fig. 6. In this case, survey of the trajectory of $\mathrm{Tl}^{3+}$ through the ionization $\mathrm{Tl}^{+}$$\mathrm{Tl}^{3+}$ as well as $\mathrm{Tl}^{2+}$ may give wider coverage of the sample volume [31], since $\mathrm{Tl}^{3+}$ has shorter gyroradus, it may more easily escape in some case the restriction of the port of the vacuum vessel. The cross-section of $\mathrm{Tl}^{+}-\mathrm{Tl}^{3+}$ has about 4-5 times smaller than that of $\mathrm{Tl}^{+}-\mathrm{Tl}^{2+}$. Fujisawa et $a l$. installed another HIBP system at different toroidal an- 


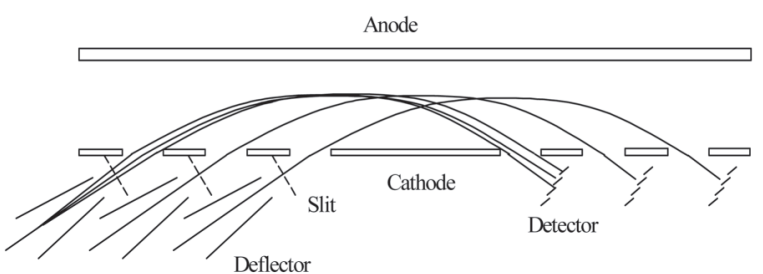

Fig. 5 Schematics of the parallel plate analyzers with as many sets of slit and detector in one analyzer.

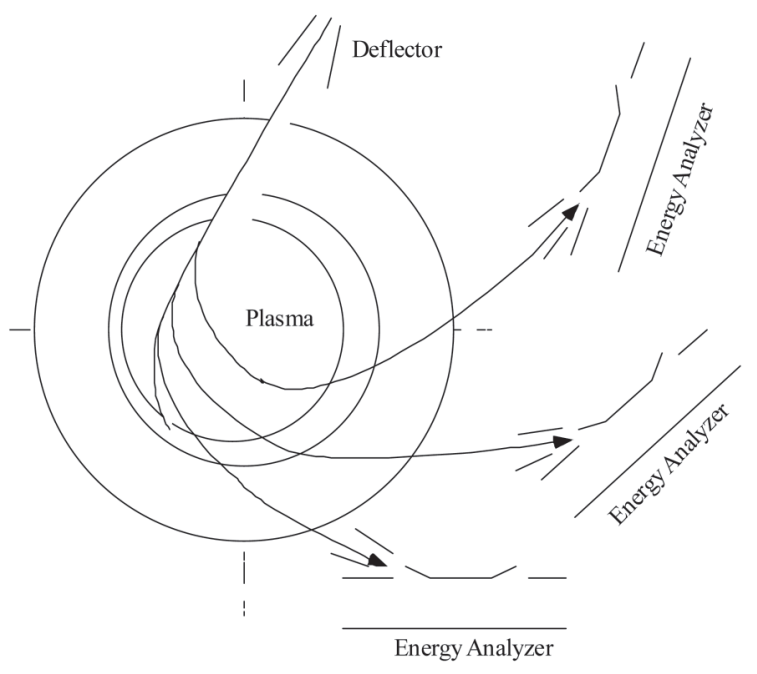

Fig. 6 Schematics of as many analyzers as possible.

gle [31]. This may give more freedom for the measurement since both are completely independent systems.

\subsection{2-D measurement of the turbulence}

In order to expand HIBP to 2-D measurement, there can be two methods. One is usage of several parallel beamlets injection, which are separated in toroidal directions and with different radial position as shown in Fig. 7.

The toroidal separation may be maintained from injection stage to analyzer stage, if it is carefully designed. By using one wide parallel plate analyzer with sets of detectors toroidally separated and sets of toroidally wide input slits, we may be able to perform 2-D turbulence measurements. Beam source and its acceleration must be done carefully, although Sony's old color TV use 3 beam system (Trinitron) and we have to study it. Another method may be a rapid sweep of the primary beam by the poloidal sweeper at the injection port shown in Fig. 1. Fast sweep of about $2-5 \mathrm{MHz}$ and the sweep voltage of $1-2 \mathrm{kV}$ in our case, may be necessary for two dimensional measurement of the drift-wave turbulence up to about $300 \mathrm{kHz}$ or so. We can test the effect of fast sweep of the primary beam by the signal due to the ionization by gas introduced into the tokamak vacuum chamber by gas puffing.

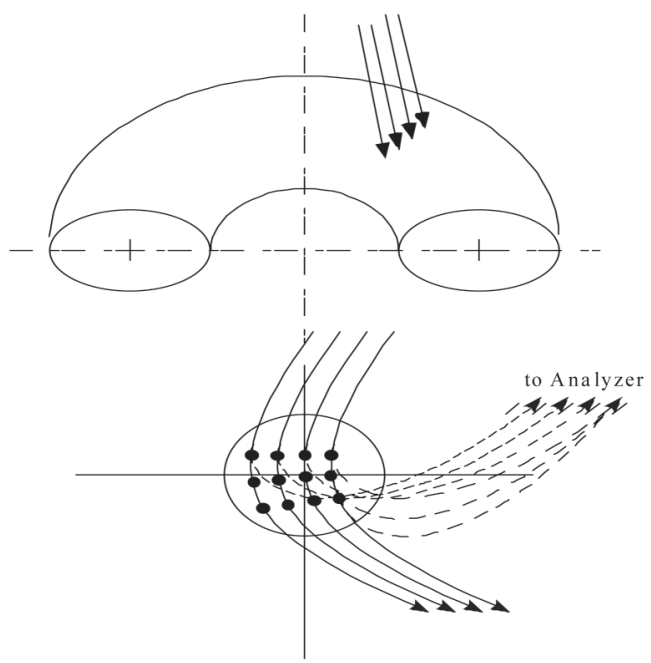

Fig. 7 Schematic for two-dimensional turbulence measurement by HIBP by poloidally and toroidally displaced injection beams.

\subsection{Beam quality}

The present thermionic ion source is very useful for obtaining smaller sample volume in the plasma. For smaller phase volume of the beam, the diameter of the ion source is very advantageous. The electron microscope use the sharp edged needle and field emission of the electron from the needle to reduce the phase volume of the beam, we have to study the field-emission-type positive ion source for electron temperature gradient drift wave turbulence, which has very high waveunumber.

\subsection{Detector development}

The injected beam should be stable and should have long-life, we should do our best to increase the sensitivity of the detector till the quantum noise (shot noise) becomes the dominant noise in the turbulence study. The most sensitive detector in the photon and beam measurement is the photo-multiplier or electron multiplier, although the direct use of the electron multiplier is difficult unless it has wide area of sensitivity.

\section{Magnetic Field Measurement}

It also may be possible to detect plasma current profile in the tokamak plasma much faster than MSE. The potential measurement of HIBP depends on the total energy conservation law in the electrostatic and magnetic field, while we may be able to measue the magnetic flux function, $R A_{\phi}$ through the by the angular canonical conservation law, $P_{\phi}=\mathrm{m} R^{2}(\mathrm{~d} \phi / \mathrm{d} t)+(e / c) R A_{\phi}$. The difficulty is this conservation holds inside the plasma where toroidal magnetic field ripple is small, while the beam detected go out of the region to the energy analyzer where the ripple is almost $100 \%$. Then we have to make a tedius calculation of the beam trajectory to the analyzer in order to interpret 
the result.

\section{Penetration of the Probing Beam}

When the plasma density increases, the most serious problem of the HIBP is the beam attenuation by the plasma electron and shortness of penetration depth of the primary beam. In JIPPT-IIU case, the highest average density for potential turbulence study is about $3 \times 10^{13} / \mathrm{cm}^{3}$ for thallium beam.

Figure 8(a) shows the intensity of the secondary beam at the energy analyzer at the sweep from plasma center to the surface, A6 to A1, when thallium $450 \mathrm{keV}$ beam is injected into the plasma of the average density of $1 \times$ $10^{13} / \mathrm{cm}^{3}$ at toroidal magnetic field of 3 Tesla. Near the plasma surface, intensity of the beam has a peak and tends to decay by small amount, in the core region. At the average density of $3 \times 10^{13} / \mathrm{cm}^{3}$, the decay of the intensity at the core is so strong and it becomes difficult to measure turbulence in the plasma core (A4-A6) because of small penetration length due to large ionization cross-section. Figure
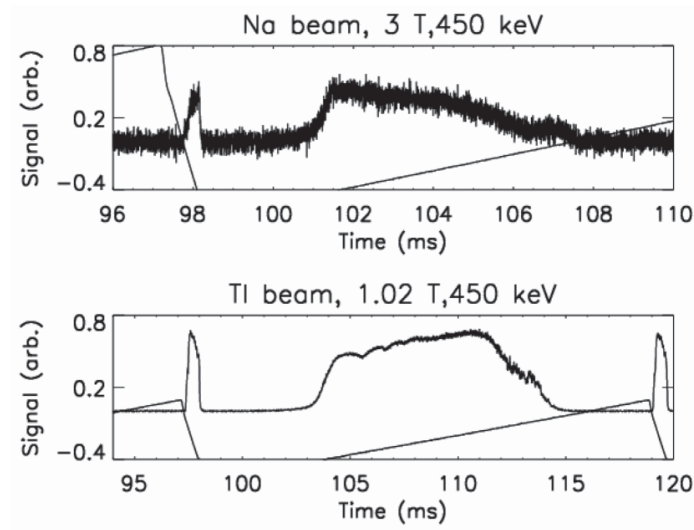

Fig. 8 The intensity of the analyzer signal at SV1 in sweep mode and poloidal sweep signal. Both at $450 \mathrm{keV}$. The average plasma density is $1 \times 10^{13} / \mathrm{cm}^{3}$. (a) thallium beam case, 3 Tesla. (b) Na beam case, 1.02 Tesla.

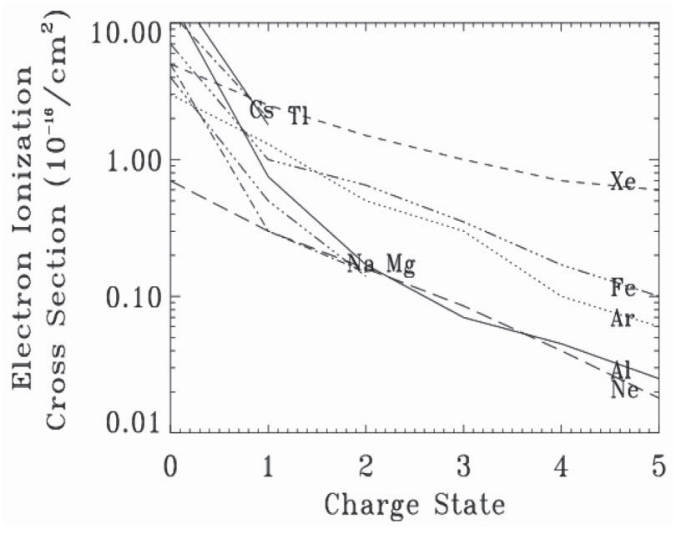

Fig. 9 Maximum electron ionization cross-section of atoms for HIBP at various ionization stage.
9 shows the maximum cross-sections by electron impact ionization of various atoms [32]. Although the penetration length depends on the dependence of cross-section on the impact electron energy, plasma temperature, maximum cross-section can be a rough measure for the selection of the atom of primary beam.

General conclusion is the usage of the beam of higher ionization state of $\mathrm{Tl}, \mathrm{Cs}$ and $\mathrm{Au}$ is not efficient for better penetration instead of usage of $\mathrm{K}$ or $\mathrm{Na}$ beam. When we changed the thallium beam to cesium, rubidium, potassium and sodium beam, the toroidal field matched to the beam, steadily goes down, and the penetration depth, however, grows steadily and the intensity profile becomes more like density profile. The tandem accelerator has another possibility for the choice of the beam with good penetration. If the negative Aluminum beam is accelerated to the central gas chamber of the tandem accelerator with the terminal voltage of $\mathrm{Va}$, it may be highly ionized to $\mathrm{Al}^{+3}$ or $\mathrm{Al}^{+4}$ with the collision of particles in the gas chamber and got the final energy of $4 \mathrm{Va}$ or $5 \mathrm{Va}$. The ionization energy of the $\mathrm{Al}^{+4}$ is more than $100 \mathrm{eV}$, compared to about $6 \mathrm{eV}$ of Thallium, the penetration depth can be so large and may be applicable to the large tokamak like JT-60U.

Figure 8(b) shows the $\mathrm{Na}^{+}$beam case instead of $\mathrm{Tl}^{+}$. Toroidal field is reduced to 1 Tesla for the identical trajectory with the case of $\mathrm{Tl}^{+}$. The intensity grows as the beam approaches the core region just like density profile, indicating much longer penetration length compared with $\mathrm{Tl}$ case.

For singly charged particle, $\mathrm{Na}$ or $\mathrm{Ne}$ beam can be candidates for more than 10 times longer penetration depth. As double charged particle, $\mathrm{Na}, \mathrm{Mg}, \mathrm{Al}$, and as triple-charged particle, $\mathrm{Mg}, \mathrm{Al}$ may be candidates.

[1] A. Hasegawa et al., Phys. Fluids, 22, 2122 (1979).

[2] A. Hasegawa and M. Wakatani, Phys. Rev. Lett. 59, 1581 (1987).

[3] J.F. Drake, P.N. Guzdar and A.B. Hassam, Phys. Rev. Lett. 6 1, 2205 (1988)

[4] R.E. Waltz, et al., Phys. Plasma 1, 2229 (1994).

[5] Z. Lin et al., Science ,281, 1835 (1998).

[6] P.H. Diamond et al., Nucl. Fusion 41, 1067 (2001).

[7] F. Jenko et al., Phys. Plasma 7, 1904 (2000).

[8] Y. Idomura et al., $20^{\text {th }}$ IAEA Fusion energy conference, Portugal, 2004.

[9] X. Garbet, Nucl. Fusion. 39, 2063 (1999).

[10] P. Beyer et al., Phys. Rev. Lett. 85, 4892 (2000).

[11] S. Benkadda et al., Nucl. Fusion. 41, 995 (2001).

[12] P.M. Schoch et al., Rev. Sci. Instrum. 59, 1646 (1988).

[13] Y. Hamada et al., Plasma Phys. Control. Fusion, 36, 1743 (1994).

[14] H.Y.W. Tsui et al., Phys. Fluids B 5, 1274 (1993).

[15] Y. Hamada et al., Fusion Eng. Des. 34-35, 663 (1997).

[16] Y. Hamada et al., Fusion Eng. Des. 34-35, 25 (1997).

[17] Y. Hamada et al., Nucl. Fusion 36, 515 (1996).

[18] B.B. Kadomtsev and O.P. Pogutse, Review of Plasma Physics, Vol.5, Consultant Bureau, Newe York, 284 (1970).

[19] P.M., Schoch et al., Rev. Sci. Instrum. 74, 1846 (2003). 
[20] G.R. McKee et al., Plasma Phys. Control. Fusion 45, A477 (2003).

[21] N. Winsor et al., Phys. Fluids 11, 2448 (1968).

[22] K. Toi et al., Plasma Physics and Controlled Nuclear Fusion Research, 1992 (Proc. 14 ${ }^{\text {th }}$ Int. Conf. Wurzburg,1992), Vol. 1, IAEA, Vienna (1993) 519.

[23] Y. Hamada et al., Nucl. Fusion 37, 999 (1997).

[24] Y. Hamada et al., Nucl. Fusion 45, 81 (2005).

[25] Y. Hamada et al., Plasma Phys. Control. Fusion 48, S177 (2006).
[26] Y. Hamada et al., Phys. Rev. Lett. 96, 115003 (2006).

[27] T.S. Hahm et al., Plasma Phys. Control. Fusion 42, A205 (2000).

[28] J.A. Boedo et al., Phys. Plasma 8, 4826 (2001).

[29] S.J. Zweben et al., Phys. Plasma 9, 1981 (2002).

[30] A. Fujisawa et al., Phys. Rev. Lett. 93, 165002 (2005).

[31] J.D. Michael et al., Rev. Sci. Instrum. 57, 1828 (1986).

[32] H. Tawara and T. Kato, Atomic and nuclear data tables 36, 167-353 (1987). 